\title{
Assessing the Cost Effectiveness of Pre-Exposure Prophylaxis for HIV Prevention in the US
}

\author{
Fred J. Hellinger
}

Published online: 24 November 2013

(C) Springer International Publishing Switzerland (outside the USA) 2013

\begin{abstract}
About 50,000 people are infected with HIV in the US each year and this number has remained virtually the same for the past decade. Yet, in the last few years, evidence from several multinational randomized clinical trials has shown that the provision of antiretroviral drug to uninfected persons (i.e. pre-exposure prophylaxis) reduces the incidence of HIV by about $50 \%$. However, evidence from cost-effectiveness studies conducted in the US yield widely varying estimates of the cost per quality-adjusted life-year (QALY) gained, and this variation reflects the substantial uncertainty surrounding the determinants of HIV transmission (e.g. adherence rates to prophylactic medications, the average number of sexual partners, the number and types of sexual acts, the viral load of infected partners, and the proportion of contacts where condoms are used), as well as different approaches to translating a reduction in HIV cases into an estimate of the increase in the number of QALYs.
\end{abstract}

\section{F. J. Hellinger $(\square)$}

Center for Delivery, Organization, and Markets, Agency for Healthcare Research and Quality (AHRQ), 540 Gaither Road, Rockville, MD 20850, USA

e-mail: fred.hellinger@ahrq.hhs.gov

\section{Key Points for Decision Makers}

- Pre-exposure prophylaxis programs have the potential to lower the cost of the HIV epidemic in the US.

- Studies of the cost effectiveness of pre-exposure prophylaxis programs should explain and justify assumptions underlying their economic model of HIV transmission.

- It is important to recognize that different assumptions about the patterns of sexual behaviour of men who have sex with men may produce very different estimates of the cost effectiveness of pre-exposure prophylaxis programs.

\section{Introduction}

The diffusion of new and powerful drugs to treat persons with HIV disease is responsible for decreases in mortality attributable to HIV and this has altered the way many people think about HIV disease. Today, HIV disease may be thought of more as a moderately expensive chronic illness than as a catastrophically expensive fatal illness [1-3]. Persons diagnosed with HIV today in the US may live almost as long as persons without HIV [4].

Yet, the progress made in the treatment of persons with HIV in the US contrasts starkly with the limited success of efforts to prevent the spread of HIV. In fact, each year about 50,000 people are newly infected with HIV in the US [5] and this number has remained relatively unchanged over the past 10 years. The number of persons who die from HIV each year in the US is about 15,000, and the number of persons living with HIV in the US has been steadily increasing and now approaches 1.2 million [6, 7]. 
Disappointing progress in slowing the spread of HIV in the US has led to calls for the implementation of new prevention strategies $[6,8]$. One of the approaches proposed by public health officials involves the use of antiretroviral therapy to reduce the infectivity of persons with HIV and to reduce the likelihood of persons without HIV acquiring HIV [9-11].

Indeed, there is some optimism that both treatment-based HIV prevention strategies (i.e. the provision of antiretroviral drugs to infected persons and the provision of antiretroviral drugs to uninfected persons) will help restrain the growth in the number of HIV infections in the US. A recent article states that "The role of antiretroviral therapy in reducing HIV incidence will probably be among the most important topics in the field of HIV prevention for years to come, and it already is being debated urgently at national and international levels within major normative agencies and charities, and by donors and implementers." [12]

In August 2011, evidence from a large, international, randomized controlled clinical trial conducted by the HIV Prevention Trials Network (HPTN) Study Team 052 on the efficacy of providing antiretroviral therapy to infected persons was published in a leading health care journal [9]. The HPTN 052 trial revealed that antiretroviral therapy for infected partners in a serodiscordant couple reduced the probability that the virus is transmitted to the uninfected partner by up to $96 \%$.

Less than a year earlier in December 2010, evidence from a large, international, randomized controlled trial, which revealed that antiretroviral therapy for uninfected men who engaged in high-risk sexual activity reduced the likelihood of infection by $44 \%$, was published in the same health care journal [13]. Since the publication of this study, results from three more large, international, randomized controlled trials have become available and each supports the finding that pre-exposure prophylaxis reduces the incidence of HIV.

Evidence from the aforementioned trials led to the approval on 16 July 2012 by the US FDA of the use of Truvada (a fixed-dose combination of tenofovir disoproxil fumarate and emtricitabine) for uninfected, high-risk individuals. In the associated boxed warning for Truvada, the FDA emphasized that pre-exposure prophylaxis with Truvada is only one part of a comprehensive prevention approach that should consist of counselling, condom use, and other safe-sex practices, along with periodic HIV testing.

At the present time there is little information available about the number of uninfected persons who are taking antiretroviral therapy. This is consequential because the cost of pre-exposure prophylaxis with Truvada is more than $\$ 10,000$ per year in the US (2010 US\$) and there are several million uninfected persons who might benefit from this course of therapy [14-19].
In order to identify cost-effectiveness studies of preexposure prophylaxis in the US, free text (cost-effectiveness analysis and HIV and prevention and Truvada OR tenofovir) was used to search PubMed/MEDLINE. About 150 articles were identified, but only four studies that included a control group, an estimate of the cost of the intervention, an estimate of the number of HIV cases averted as a result of the prophylaxis, and estimates for the cost effectiveness of this intervention were included in this review.

\section{Treatment-Based Prevention Strategies}

\subsection{Treatment of Infected Persons}

Evidence from the HPTN 052 Study Team's evaluation of the impact of the treatment of infected individuals earlier in the course of illness revealed that the probability of transferring HIV was significantly reduced [9]. The HPTN 052 randomized clinical trial examined transmission of HIV among serodiscordant couples at 13 sites in nine countries (Botswana, Kenya, Malawi, South Africa, Zimbabwe, Brazil, India, Thailand, and the US) and it compared the efficacy of antiretroviral therapy plus HIV primary care versus HIV primary care alone to prevent the sexual transmission of HIV in serodiscordant couples [9]. In this trial, one half of the infected partners in 1,763 serodiscordant couples who had CD4+ cell counts between 350 and 500 cells per cubic millimetre received immediate antiretroviral therapy, and the infected partner in the other half of the serodiscordant couples who also had CD4+ cell counts between 350 and 500 cells per cubic millimetre did not receive antiretroviral therapy.

This study found that of the 28 virologically linked HIV transmissions observed during the study period among participants in HPTN 052, only one occurred in the treatment arm and the remaining 27 occurred in the control group. Yet, since guidelines for the commencement of antiretroviral therapy in the US already suggest that early treatment be considered for all infected patients, the findings from HPTN 052 were not expected to have a significant impact on HIV incidence rates.

\subsection{Treatment of Uninfected Persons}

Treatment-based HIV prevention involving uninfected persons includes treatment of uninfected persons immediately after exposure to HIV (post-exposure prophylaxis) as well as the treatment of uninfected persons before exposure to HIV (pre-exposure prophylaxis). While post-exposure prophylaxis has been accepted treatment for accidental exposure to HIV from needle sticks for many years, pre-exposure prophylaxis is a relatively new strategy for HIV prevention. 
Yet, pre-exposure prophylaxis is not a new strategy for the prevention of a number of other diseases. For example, individuals who are planning to travel to countries where malaria and tuberculosis are common are often given drugs to protect them from getting these diseases. In particular, most US citizens who travel to areas with a high prevalence of malaria and tuberculosis are told to take prophylactic drugs, and the drugs used to prevent malaria and tuberculosis are generally the same drugs that are used to treat these disease (chloroquine tablets for malaria, and isoniazid tablets for tuberculosis).

Interest in pre-exposure prophylaxis for HIV has grown since findings from a number of randomized clinical trials have shown that persons without HIV who receive antiretroviral drugs (specifically tenofovir disoproxil fumarate and/or emtricitabine) are less likely to become infected than persons who do not receive these drugs [20-22].

This study briefly reviews randomized controlled trials where the treatment group includes uninfected individuals who receive antiretroviral drugs and where the control group includes uninfected individuals who do not receive antiretroviral drugs (see Table 1). Each of the studies includes information about the number of subjects who became infected over a specific period of time and the results of each of these studies has been published in a peer reviewed medical journal. Although this manuscript does not review each and every effort to prevent HIV infection by providing uninfected individuals with antiretroviral drugs, it does review the largest and most influential studies of pre-exposure prophylaxis against HIV infection.

One of the earliest trials of the efficacy of pre-exposure prophylaxis involved testing the use of tenofovir disoproxil fumarate as a microbicide gel [20]. In this trial [the Centre for the AIDS Program of Research in South Africa (CAPRISA) 004 trial], 1085 women were enrolled and randomly assigned in equal proportions to the treatment and placebo groups between May 2007 and January 2009. This study examined a coitally-related strategy where doses were applied both before and after sexual contact. The tenofovir disoproxil fumarate and placebo gels were packaged in the same type of vaginal applicators and were indistinguishable. Women were followed at monthly visits for 30 months and tenofovir disoproxil fumarate gel reduced acquisition by $39 \%$ overall (HIV incidence was 5.6 per 100 women-years for the treatment group and 9.1 per 100 women-years in the placebo group). Efficacy was

Table 1 Trials of pre-exposure prophylaxis with antiretroviral drugs

\begin{tabular}{|c|c|c|c|c|c|}
\hline Trial name & $\begin{array}{l}\text { High-risk } \\
\text { population }\end{array}$ & Location & Drug & $\begin{array}{l}N \text { (enrollment } \\
\text { dates) }\end{array}$ & Results \\
\hline iPrEx [13] & $\begin{array}{l}\text { MSM and } \\
\text { transgender } \\
\text { women }\end{array}$ & $\begin{array}{l}\text { Brazil, Ecuador, } \\
\text { Peru, South } \\
\text { Africa, } \\
\text { Thailand, US }\end{array}$ & Oral TDF-FTC & $\begin{array}{l}\text { 2,499 (July } \\
\text { 2007-Dec } \\
\text { 2009) }\end{array}$ & $\begin{array}{l}\text { Tenofovir and emtricitabine } \\
\text { reduced incidence by } 44 \%\end{array}$ \\
\hline CAPRISA [20] & $\begin{array}{l}\text { Heterosexual } \\
\text { women }\end{array}$ & South Africa & $\begin{array}{l}\text { Topical vaginal } \\
\text { TFV }\end{array}$ & $\begin{array}{l}\text { 1,085 (May } \\
\text { 2007-Jan } \\
\text { 2009) }\end{array}$ & $\begin{array}{c}\text { Tenofovir gel reduced } \\
\text { acquisition by } 39 \%\end{array}$ \\
\hline FEM-PrEP [23] & $\begin{array}{l}\text { Heterosexual } \\
\text { women }\end{array}$ & $\begin{array}{l}\text { Kenya, South } \\
\text { Africa, } \\
\text { Tanzania }\end{array}$ & $\begin{array}{l}\text { Daily oral TDF- } \\
\text { FTC }\end{array}$ & $\begin{array}{l}\text { 2,120 (June } \\
\text { 2009-April } \\
\text { 2011) }\end{array}$ & $\begin{array}{l}\text { Trial was terminated because } \\
\text { there was not a significant } \\
\text { difference in the number of } \\
\text { HIV infections }\end{array}$ \\
\hline TDF2 [22] & $\begin{array}{l}\text { Heterosexual } \\
\text { men and } \\
\text { women }\end{array}$ & Botswana & $\begin{array}{l}\text { Daily oral TDF- } \\
\text { FTC }\end{array}$ & $\begin{array}{l}\text { 1,219 (March } \\
\text { 2007-May } \\
\text { 2010) }\end{array}$ & $\begin{array}{l}\text { Efficacy of tenofovir and } \\
\text { emtricitabine was } 62.2 \%\end{array}$ \\
\hline VOICE [24] & $\begin{array}{l}\text { Heterosexual } \\
\text { women }\end{array}$ & $\begin{array}{l}\text { Uganda, South } \\
\text { Africa, and } \\
\text { Zimbabwe }\end{array}$ & $\begin{array}{l}\text { Oral TDF-FTC } \\
\text { and vaginal } \\
\text { microbicide }\end{array}$ & $\begin{array}{l}\text { 5,029 (Sept } \\
\text { 2009-Aug } \\
\text { 2012) }\end{array}$ & $\begin{array}{l}\text { Neither oral tenofovir tablets } \\
\text { nor tenofovir gel were } \\
\text { effective in preventing HIV } \\
\text { among the women in the } \\
\text { study }\end{array}$ \\
\hline Partners PrEP [21] & $\begin{array}{l}\text { Serodiscordant } \\
\text { heterosexual } \\
\text { couples }\end{array}$ & Kenya, Uganda & $\begin{array}{l}\text { Daily oral } \\
\text { tenofovir or } \\
\text { TDF-FTC }\end{array}$ & $\begin{array}{l}4,747 \text { (July } \\
2008-\text { Nov } \\
\text { 2010) }\end{array}$ & $\begin{array}{l}75 \% \text { reduction in incidence } \\
\text { among the tenofovir and } \\
\text { emtricitabine group; } 67 \% \\
\text { reduction in tenofovir only } \\
\text { group }\end{array}$ \\
\hline
\end{tabular}

iPrEx Pre-Exposure Prophylaxis Initiative, CAPRISA Centre for the AIDS Program of Research in South Africa, FEM-PrEP Pre-exposure Prophylaxis Trial for HIV Prevention among African Women, VOICE Vaginal and Oral Interventions to Control the Epidemic, MSM men who have sex with men, TDF-FTC tenofovir disoproxil fumarate-emtricitabine, $\operatorname{PrEP}$ pre-exposure prophylaxis 
closely related to adherence, with high adherers (defined as more than $80 \%$ ) experiencing $54 \%$, and low adherers (defined as $<50 \%$ ) experiencing $28 \%$.

In another early trial of pre-exposure prophylaxis (the Pre-Exposure Prophylaxis Initiative-iPrEx), clinicians screened 4,900 subjects for inclusion between July 2007 and December 2009 [13]. Participants in iPrEx included men who have sex with men (MSM) and transgender women who were being treated at 11 sites in six countries (Peru, Ecuador, South Africa, Brazil, Thailand, and the US). This trial was supported by the National Institute of Allergy and Infectious Diseases (NIAID) of the National Institutes of Health, and by the Bill and Melinda Gates Foundation. The trial included 2,400 participants, with half of those receiving Truvada and the remaining half a placebo. Of the 100 subjects who seroconverted during this trial, only 36 were in the tenofovir disoproxil fumarate and emtricitabine group and the remaining 64 in the placebo group. This indicates a $44 \%$ reduction in the incidence of $\mathrm{HIV}$ in the tenofovir disoproxil fumarate and emtricitabine group.

In a third randomized clinical trial of pre-exposure prophylaxis (the Partners Pre-exposure Prophylaxis study), 1474 HIV serodiscordant heterosexual couples from Kenya and Uganda were randomized into three groups between July 2008 and November 2010 [21]. The first group received once-daily tenofovir disoproxil fumarate. The second group received daily tenofovir disoproxil fumarate and emtricitabine (i.e. Truvada). The third group did not receive either. Of the 82 infections that occurred among the seronegative partners in this study, only 13 occurred in the tenofovir disoproxil fumarate and emtricitabine group, 17 occurred in the tenofovir disoproxil fumarate group, and the remaining 52 infections occurred in the placebo group. This indicates that the relative reduction was $75 \%$ in incidence among the tenofovir disoproxil fumarate and emtricitabine group and $67 \%$ reduction in incidence among the tenofovir disoproxil fumarate-only group. Adherence among those in the treatment group who seroconverted during the trial was low (31\% had detectable tenofovir disoproxil fumarate levels in a plasma sample) compared with adherence among those in the treatment group who did not seroconvert during the trial $(82 \%$ of this group had detectable tenofovir disoproxil fumarate levels). When comparing those who had a detectable level of tenofovir disoproxil fumarate in their plasma to those who did not, the estimated reduction in the relative risk of seroconverting during the trial was $86 \%$ for the tenofovir disoproxil fumarate group and $90 \%$ lower for the tenofovir disoproxil fumarate and emtricitabine group. This trial was funded by the Bill and Melinda Gates Foundation

In a fourth trial (the TDF2 study), 1219 seronegative men and women in the Botswana cities of Francistown and Gaborone (Botswana has the world's second highest prevalence of HIV) were randomly assigned to receive tenofovir disoproxil fumarate and emtricitabine or a matching placebo once daily [22]. The first participants in the TDF2 trial were enrolled in March 2007 and the last were enrolled in October 2009. All enrollees exited the trial by the end of May 2010, and at this time there were 33 participants who had become infected; nine were in the treatment group and 24 in the placebo group. There were 1.2 and 3.1 infections per 100 person-years, respectively, in each group, indicating that the efficacy of tenofovir disoproxil fumarate and emtricitabine was $62.2 \%$. The authors concluded that tenofovir disoproxil fumarate and emtricitabine prophylaxis prevented HIV infection in sexually active heterosexual adults.

Not all randomized clinical trials have found pre-exposure prophylaxis with antiretroviral drugs to be effective in the prevention of HIV infection. In fact, a recent randomized, double-blind, placebo-controlled trial of HIV negative women aged 18-35 years in Kenya, South Africa, and Tanzania [the Pre-exposure Prophylaxis Trial for HIV Prevention among African Women (FEM-PrEP)] was terminated early because of a lack of efficacy [23]. Between June 2009 and April 2011, the FEM-PrEP trial randomly assigned $2120 \mathrm{HIV}$-negative women to receive either a combination of tenofovir disoproxil fumarate and emtricitabine or a placebo once daily. At the time it was discontinued, there was not a significant difference in the number of HIV infections between the two groups (a total of 68 women had seroconverted-33 of these were in the treatment group and 35 in the placebo group). This study also found that more women discontinued therapy in the treatment group due to hepatic and renal abnormalities, and that more women in the treatment group experienced nausea, vomiting and elevated alanine aminotransferase levels.

Just as in other pre-exposure prophylaxis trials, the FEM-PrEP included extensive educational activities designed to promote adherence. Yet, just as in the other trials, low levels of adherence were a problem. In fact, drug-level testing revealed that only $26 \%$ of treated participants who seroconverted had positive results for the existence of tenofovir disoproxil fumarate in their plasma at the beginning of the infection window and $21 \%$ had positive results for the existence of tenofovir disoproxil fumarate at the end of the window, while only $15 \%$ were positive for the existence of tenofovir disoproxil fumarate at both time periods. These findings belie the accuracy of self-reported data about adherence because $95 \%$ of women indicated that they were compliant with the drug regimen when asked about adherence in a survey conducted at the discontinuation of the trial. 
A recent randomized clinical trial whose results are still being analyzed is the Vaginal and Oral Interventions to Control the Epidemic (VOICE) trial [24-26]. This trial was conducted by researchers in the Microbicide Trials Network, which is funded by the NIAID, the National Institute of Child Health and Human Development, and the National Institute of Mental Health. The VOICE trial conducted a study of the daily use of tenofovir disoproxil fumarate gel and oral tenofovir disoproxil fumarate tablets on the incidence of HIV infection among 5,029 women in Uganda, South Africa, and Zimbabwe who were enrolled in this study, beginning in September 2009.

The tenofovir disoproxil fumarate gel arm of the VOICE trial was discontinued in September 2011 due to the lack of evidence that the intervention was reducing the incidence of HIV infection (e.g. the HIV incidence rate in the placebo group of the tenofovir disoproxil fumarate gel arm was 6.1 per 100 women-years, while the HIV incidence rate of the treatment arm was 6.0 per 100 women-years), and the tenofovir disoproxil fumarate tablet arm of the VOICE trial was discontinued in November 2011 for the same reason. Yet, even though self-reported adherence rates were quite high for all participants in the VOICE trial, it is certainly possible that these rates were overstated by the participants. Indeed, the congruence between self-reported adherence rates and actual adherence rates in studies of pre-exposure prophylaxis for HIV infections has been relatively low (note, some studies conduct laboratory tests to determine whether or not participants are taking the recommended medications).

\section{Models of HIV Transmission}

One of the challenges facing health care decision makers is how to reduce the number of persons who are infected with HIV each year. Efforts to devise a successful strategy must target those communities most deeply affected by this epidemic, and this is especially difficult because these communities are often in the poorest and most neglected sections of our large cities. Furthermore, the extended and unpredictable time period between exposure to HIV and when symptoms and signs are initially evident, coupled with the low probability of transmission due to a single contact, mean that the composition and diversity of the targeted population are key variables in determining how to organize a campaign to slow the spread of the virus.

Analysts construct mathematical models based on the underlying transmission mechanisms of the HIV virus to help them predict the effectiveness of various strategies to slow the spread of this virus. The more accurate and robust the model, the better able analysts are to design an effective strategy to slow the spread of the virus.
Stanley [27] stated that,

Mathematical modelling studies have shown that the AIDS epidemic is very sensitive to the human behaviours that spread HIV, including: the amount of risky behaviour; the manner in which that risky behaviour is distributed in the population; and the social network structures within which people practice those risky behaviours.

Individual risk-based models of the dynamics of the spread of HIV infection require data on the behavioural patterns of MSM and injection drug users [28]. In particular, these models require estimates of the number of new partners per year, the number and type of sexual contacts, the viral load of each infected partner, the proportion of sexual contacts where a condom was used, the number of instances of needle sharing, and the probability of infection from a single sexual contact or a single instance of needle sharing.

The accuracy of individual risk-based models depends on the validity of the assumptions about risky behaviour. For example, in a recent study Juusola and colleagues [29] assumed that there are 6.4 million MSM aged 13-64 years in the US, that the average number of partners per MSM per year is three, that a condom is used with $40 \%$ of partners, and that the annual transmission rate per MSM partnership is 0.21 when the infected partner is in the acute phase, 0.039 in the asymptomatic phase, 0.039 in the symptomatic phase, and 0.16 when the infected partner has AIDS. Juusola and colleagues [29] also assumed that there is no serosorting (i.e. proportional mixing), which implies that men do not alter their behaviour in response to the perceived serostatus of their partner. Each of these assumptions is important and each involves substantial uncertainty.

Nevertheless, useful insights may still be gleaned from individual risk-based models of HIV transmission. Indeed, the growth of qualitative research attests to the need to define questions and offer answers to questions that are not easily formulated, quantified, or solved using convenient mathematical solutions. Researchers who model HIV transmission have been able to provide insights into the factors that explain why the epidemic has diffused in a specific manner. For example, modellers have shown that if there is no serosorting and individuals seek partners without regard to their risk-taking behaviour then the spread of the HIV epidemic among low-risk users should be quite rapid even in the early stages of the epidemic [30]. Alternatively, if there is relatively little contact between highrisk and low-risk individuals then the spread of the epidemic among high-risk users should be quite high when compared with the spread among low-risk users. Indeed, the latter is true, which suggests that the amount of sexual 
contact and needle-sharing between the relatively small groups of high-activity individuals and the larger group of low-activity individuals is limited.

This feature of the epidemic requires models where partners are not chosen proportionally but are chosen based on the similarity of demographic and behavioural characteristics. Yet, biased mixing models require detailed information about the behavioural patterns of potential partners and this type of information about social networks is generally unavailable. They also require information on racial and ethnic characteristics of persons who engage in risky behaviour. Again, this type of information is normally unavailable to model builders.

Another important feature of the epidemic relates to the age and sex of persons who become infected through homosexual sexual acts, heterosexual sexual acts, and needle sharing. In general, heterosexual women who become infected are younger than their male partners, and this suggests that the age structure of heterosexual women who become infected in an area is younger than for the heterosexual men who become infected. Furthermore, in many areas there is little mixing between MSM and injection drug users. Thus, modellers may treat the epidemic in homosexual men and injection drug users as separate epidemics because although it is possible for persons to engage in both types of risk behaviour and to interact with both homosexual men and injection drug users, research suggests that the practical consequences of HIV transmission by homosexual injection drug users is nominal. Researchers have also concluded that it is unlikely that a primary epidemic in one of these risk categories could maintain a secondary HIV epidemic in the other risk category as a result of the activities of homosexual drug users.

HIV disease transmission models often explain the diffusion of HIV by defining a set of equations that specify the movement of individuals through a particular array of health states that include an uninfected state (often referred to as a susceptible state) and an infected state. These models frequently use differential equations where the dependent variable of interest is the transmission of HIV [31-34]. In particular, these models delineate the likelihood of an individual with a specific set of demographic and behavioural characteristics of engaging in risky behaviour and of becoming infected.

The probability that a person moves from one state to another is often modelled using difference or differential equations. Since HIV transmission models involve products of the number of susceptible individuals and the number of infected individuals, these equations are almost always non-linear. Yet, only the simplest differential equations may be solved explicitly with a self-contained formula so analysts normally rely on numerical analysis, and in most instances this involves the use of approximation methods that express the solution in terms of infinite series.

Because individual risk-based models of HIV transmission require a great deal of information about the nature, frequency, and characteristics of sexual activity of MSM, modellers must make strong assumptions. The difficulty in verifying these assumptions and the absence of information about many of the important variables related to the sexual activities of individuals has limited the value of individual risk-based HIV transmission models in forecasting the course of the HIV epidemic.

If at all possible, individual risk-based models of HIV transmission should be corroborated before they are utilized, to predict the number of new HIV infections in a given population. Yet, individual-based risk models of HIV transmission are difficult to corroborate and few attempts have been made to utilize these models to estimate the number of new HIV infections for large populations. Accordingly, individual risk-based transmission models have not been shown to be particularly helpful in forecasting HIV incidence in the US or even for regions of the US, and virtually all of the models used to forecast the number of new HIV infections in the US are based on straightforward exponential or polynomial extrapolations of current trends in the relevant population.

Indeed, extrapolation models represent the most common and most clear-cut means of predicting the number of future cases of HIV infection. Most often modellers simply fit existing incidence data to an assumed form for the incidence curve and then lengthen this curve to predict the number of future HIV cases. The obvious benefits of extrapolation techniques are its straightforwardness and its ease of use. Modellers may utilize this technique to estimate future HIV incidence without making specific assumptions about the nature and frequency of sexual contacts about populations with a high risk of contracting HIV.

One of the major limitations of population-based HIV forecasting models is the assumption that the process which determines the number of new HIV infections does not change through time. This assumption may be defensible if predictions are made for short time periods but it is clearly untenable when used to predict future activity many years into the future.

Moreover, population-based extrapolation models are not especially useful in assessing the importance of prevention strategies because they do not replicate the transmission processes of HIV. Manifestly, such models are useful over the short term but are incapable of providing information about the impact of changes in the demographic characteristics and behavioural patterns of the relevant populations. Consequently, they are unable to 
elucidate how the epidemic will diffuse through various population groups and how new medical treatments, changing behavioural patterns, and increased access to antiretroviral therapies will alter these diffusion patterns.

\section{HIV Disease Transition Models}

After an individual is infected with HIV, analysts may employ disease transition models to study the clinical evolution of HIV [35-37]. Most HIV disease transition models define a number of health states and specify the probability of persons to remain in a given health state or transition forward to the next health state.

These models generally define a large number of health states using CD4 cell counts, HIV RNA levels (i.e. measures of viral load), and the patient's history of clinical events, and then specify the probability of moving from one state to another. These models customarily assume that the probability of clinical progression depends only on the health state and not on the patient's previous history (this assumption is referred to as the Markovian assumption and these models are referred to as Markov models) [38, 39].

A large number of health states are generally defined in these models because patients with different clinical and demographic characteristics often have distinct prognoses. For example, the indefinite persistence of drug-resistant variants in resting CD4-positive $\mathrm{T}$ cells, monocytes, macrophages, and other cells suggests that patients with such variants may have different clinical prognoses than patients without such variants [31, 40, 41]. Furthermore, there is evidence that the nadir CD4-positive T-cell count predicts a patient's response to future therapies, suggesting that patients should be grouped according to this variable [42], and there is evidence that patients with multidrug-resistant HIV-1 infection have a poorer prognosis than patients without drug-resistant HIV-1 infection [43, 44]. There is also evidence indicating that initial HIV-1 RNA levels, a history of substance abuse, age, and drug metabolism affect the clinical outcome of drug therapy.

Yet, analysts generally do not create categories based on the existence of the resistance characteristics in patients before therapy, the nadir CD4-positive T-cell count, the existence of multidrug-resistant $\mathrm{HIV}-1$ infection under therapy, initial HIV-1 RNA level, a history of substance abuse, age, and drug metabolism because data on all these characteristics of patients are often unavailable and it would be an exceedingly difficult task to assign progression probabilities to each of the stages defined by these variables. Consequently, analysts must make simplifying assumptions in order to reduce the number of disease stages to a manageable number.

\section{Cost-Effectiveness Analysis}

It is important for planners and policy makers to have good information about the cost of the health care services utilized by persons with HIV disease. The Federal government spent more than $\$ 28$ billion in the fiscal year 2012 (2012 US\$) on assistance to persons with HIV disease, and most persons with HIV disease in the US depend upon public sources to pay for services [45, 46]. The bulk of these funds were spent on providing health care services, and, in order to ensure that all patients have access to appropriate services, planners and policy makers must have information about the cost effectiveness of various prevention and treatment strategies.

Estimates of the cost effectiveness of pre-exposure prophylaxis for HIV infection are derived by combining findings from epidemiological models of the dynamics of HIV transmission, findings from models of the transition of infected individuals through a series of health states, and findings from economic models. Information about the cost of resources used by uninfected individuals who are taking antiretroviral drugs (e.g. prophylactic drugs, HIV testing, HIV counselling, physician services, outpatient facility charges, and a variety of laboratory procedures) and about the cost of resources used by infected individuals in a given health state enable analysts to estimate the cost effectiveness of pre-exposure prophylaxis.

Defining an economic model of HIV disease as one which measures the economic value of the resources consumed in the care of a person with HIV disease, researchers have used economic models of HIV disease to examine a myriad of issues. For example, economic models have been used to compare the desirability of alternative interventions to prevent the spread of HIV disease as well as to compare the desirability of alternative treatment regimens. In most instances, the desirability of interventions and treatments has been measured using cost-effectiveness analyses.

Cost-effectiveness analysis may be used to measure the cost per unit of effect for HIV pre-exposure prophylaxis prevention interventions. For prevention interventions, the unit of effect may be the number of HIV cases averted or the number of quality-adjusted life-years (QALYs) gained. Whether a specific value for the cost per unit of effect is cost effective is related to the frame of reference of the observer. What is cost effective in the US may not be cost effective in a nation with more limited resources. For example, two studies found that the cost per QALY for routine screening for HIV infection in the US was about $\$ 40,000$ (US\$), which was below the common threshold of $\$ 50,000$ per QALY for interventions in the US but considerably higher than common thresholds in less developed nations $[47,48]$. 
Before the diffusion of highly active antiretroviral therapy in 1996 there were relatively few drug combinations used to treat persons with HIV disease and the impact on survival of the various NRTI drug regimens was comparable. Drug therapy often involved only one medication, and patients generally stayed on the same drug regimen unless they developed a serious adverse reaction. As a result, the economic models used to estimate the annual and lifetime costs of therapy during this time were relatively simple.

Economic models of HIV therapy join estimates of the cost of treating HIV patients in a number of disease stages (usually defined using CD4 cell counts and AIDS status) with estimates of the average time spent in each stage. The estimates for the cost of care in a given disease stage are derived by multiplying the number of hospital days, outpatient visits, drugs, and other resources (e.g. home health visits, days of long-term care) by estimates of the unit cost of each of these services. The costs across stages are then summed to obtain an estimate of the yearly or lifetime cost of care.

\section{Cost-Effectiveness Studies of Pre-Exposure Prophylaxis}

Cost-effectiveness studies reviewed in this manuscript were identified by screening the PubMed/MEDLINE database for several phrases (cost-effectiveness analysis and HIV and prevention and Truvada OR tenofovir disoproxil fumarate). In addition, one study of the cost effectiveness of sexually active women in South Africa [49] was included because it was rigorously constructed, widely cited, and published in a peer reviewed journal.

The cost effectiveness of pre-exposure prophylaxis for HIV infection studies reviewed in this manuscript have been published between 2008 and 2012 (see Table 2). Yet, findings from these studies are inconsistent and it is important for those who rely on these models to understand the reasons for the wide variation in estimates of the cost per QALY gained.

A 2008 study of pre-exposure prophylaxis by Desai and colleagues [50] calculated that the incremental cost per QALY gained in the US was \$31,970 (2007 US\$), while a year later Paltiel and colleagues [51] calculated that the incremental cost per QALY saved was \$298,000 (2006 US\$). Both of these studies used HIV transmission and progression models to estimate the cost of reducing the number of new HIV infections, and both used published estimates of the cost of HIV treatment to generate their estimates of the incremental cost of a QALY.

The study by Desai and colleagues assessed the cost effectiveness of a 5-year program of chemoprophylaxis and they found that more than one-half of the number of HIV infections averted was not among those who were taking prophylactic drugs but among those who benefited from reduced prevalence in the target population. They converted the decrease in the number of HIV infections to an

Table 2 Cost-effectiveness studies of pre-exposure prophylaxis with antiretroviral drugs

\begin{tabular}{|c|c|c|c|c|c|c|}
\hline References & $\begin{array}{l}\text { Target } \\
\text { population }\end{array}$ & Drug & Approach & $\begin{array}{l}\text { Prevalence/ } \\
\text { incidence }\end{array}$ & Effectiveness & $\begin{array}{l}\text { Cost per } \\
\text { QALY }\end{array}$ \\
\hline $\begin{array}{l}\text { Desai et al. } \\
\text { [50] }\end{array}$ & $\begin{array}{l}\text { High-risk } \\
\text { MSM in } \\
\text { the US }\end{array}$ & $\begin{array}{l}\text { Oral TDF- } \\
\text { FTC }\end{array}$ & $\begin{array}{l}\text { Uses population-based HIV } \\
\text { transmission dynamic model }\end{array}$ & $\begin{array}{l}\text { Prevalence is } \\
14.6 \%\end{array}$ & $\begin{array}{l}\text { Assumes PrEP reduces } \\
\text { HIV transmission } \\
\text { probabilities by between } \\
50 \text { and } 70 \%\end{array}$ & $\begin{array}{l}\$ 31,970 \\
(2007 \\
\text { US\$) }\end{array}$ \\
\hline $\begin{array}{l}\text { Paltiel et al. } \\
\text { [51] }\end{array}$ & $\begin{array}{l}\text { High-risk } \\
\text { MSM in } \\
\text { the US }\end{array}$ & $\begin{array}{l}\text { Oral TDF- } \\
\text { FTC }\end{array}$ & $\begin{array}{l}\text { Uses individual-level state } \\
\text { transition Monte Carlo } \\
\text { simulation model of HIV } \\
\text { acquisition }\end{array}$ & $\begin{array}{l}\text { MSM population } \\
\text { with } 1.6 \% \\
\text { annual HIV } \\
\text { incidence rate }\end{array}$ & $\begin{array}{l}\text { Assumes PrEP reduces } \\
\text { HIV transmission rate by } \\
50 \%\end{array}$ & $\begin{array}{l}\$ 298,000 \\
(2006 \\
\text { US\$) }\end{array}$ \\
\hline $\begin{array}{l}\text { Walensky } \\
\text { et al. [49] }\end{array}$ & $\begin{array}{l}\text { South } \\
\text { African } \\
\text { women }\end{array}$ & $\begin{array}{l}\text { Tenofovir- } \\
\text { based } \\
\text { vaginal } \\
\text { microbicide }\end{array}$ & $\begin{array}{l}\text { Uses individual-level state } \\
\text { transition Monte Carlo } \\
\text { simulation model of HIV } \\
\text { acquisition }\end{array}$ & $\begin{array}{l}\text { Annual incidence } \\
\text { is } 2.2 \%\end{array}$ & $\begin{array}{l}\text { Assumes PrEP reduces risk } \\
\text { by } 39 \%\end{array}$ & $\begin{array}{l}\$ 2,700 \\
(2010 \\
\text { US\$) }\end{array}$ \\
\hline $\begin{array}{l}\text { Juusola et al. } \\
\text { [53] }\end{array}$ & $\begin{array}{l}\text { MSM in } \\
\text { the US }\end{array}$ & $\begin{array}{l}\text { Oral TDF- } \\
\text { FTC }\end{array}$ & $\begin{array}{l}\text { Uses risk-based dynamic } \\
\text { compartmental model of HIV } \\
\text { transmission }\end{array}$ & $\begin{array}{l}\text { Annual incidence } \\
\text { is .8\% }\end{array}$ & $\begin{array}{l}\text { Assumes } 44 \% \text { reduction } \\
\text { in risk due to PrEP }\end{array}$ & $\begin{array}{l}\$ 172,000 \\
(2009 \\
\text { US\$) }\end{array}$ \\
\hline $\begin{array}{l}\text { Koppenhaver } \\
\text { et al. [54] }\end{array}$ & $\begin{array}{l}\text { Urban } \\
\text { MSM in } \\
\text { the US }\end{array}$ & $\begin{array}{l}\text { Oral TDF- } \\
\text { FTC }\end{array}$ & $\begin{array}{l}\text { Uses a dynamic compartmental } \\
\text { HIV transmission simulation } \\
\text { model for MSM in an urban } \\
\text { community }\end{array}$ & $\begin{array}{l}\text { Prevalence is } \\
17.5 \%\end{array}$ & $\begin{array}{l}\text { Assumes } 44 \% \text { reduction } \\
\text { in risk due to PrEP }\end{array}$ & $\begin{array}{l}\$ 31,970 \\
(2010 \\
\text { US } \$)\end{array}$ \\
\hline
\end{tabular}

$M S M$ men who have sex with men, $T D F-F T C$ tenofovir disoproxil fumarate-emtricitabine, $\operatorname{PrEP}$ pre-exposure prophylaxis, $Q A L Y$ qualityadjusted life-year 
estimate of the incremental cost per QALY using a basecase HIV-related lifetime treatment cost of US\$343,130 (2007 US\$) and an estimate of 6.95 QALYs saved per case of HIV prevented [50].

Paltiel and colleagues [51] used an individual level model to simulate the impact of pre-exposure prophylaxis on the incidence of HIV infection, and they assumed that pre-exposure prophylaxis lowers age-specific incidence rates by $50 \%$. They derived their estimate of $\$ 298,000$ (2006 US\$) per QALY by transforming clinical and economic data in their population-based HIV disease statetransition model, referred to as the Cost-Effectiveness of Preventing AIDS Complications (CEPAC) model [52].

Paltiel and colleagues [51] generated estimates of the costs of treatment and measures of the outcomes of particular treatment regimens. Their study estimated that preexposure prophylaxis would reduce the lifetime HIV infection risk from 44 to $25 \%$ (under the base-case assumption of $50 \%$ efficacy in a population with a $1.6 \%$ mean annual incidence of HIV infection) while increasing mean life expectancy by 1.7 years (39.0-40.7) and lifetime treatment costs from $\$ 81,000$ to $\$ 252,700$ per person (2006 US $\$$ ).

Juusola and colleagues [53] hypothesized that the efficacy of pre-exposure prophylaxis was $44 \%$ (based on findings from the iPrEx trial) and then calculated the cost effectiveness of a pre-exposure prophylaxis program that treated $20 \%$ of MSM in the US. The authors used a dynamic model of HIV treatment and progression and found that the pre-exposure prophylaxis program reduced the number of new HIV infections by $13 \%$ and increased the number of QALYs by 550,000 at a cost of $\$ 172,000$ per QALY (2010 US\$). They also calculated that 62,800 cases of HIV would be prevented and that the program would cost $\$ 98$ billion (2010 US\$).

The authors also noted that the cost effectiveness of an HIV pre-exposure prophylaxis program could be increased significantly if it could be initiated for only high-risk MSM. This observation is true for all pre-exposure prophylaxis programs. However, restricting the availability of prophylactic drugs to only those who are at the highest risk for becoming infected may not be possible because the information necessary to identify such individuals is difficult to obtain and because all those at risk have an incentive to participate in the program.

In a recent study, Walensky and colleagues [49] employed a sophisticated, well-documented, and widely referenced HIV disease transition model, referred to as the CEPAC-international model, to estimate the cost effectiveness of pre-exposure prophylaxis using a tenofovir disoproxil fumarate-based vaginal microbicide among heterosexual South African women. The CEPAC-international model is a dynamic, compartmental, health state transition model that follows persons (index patients) from the occasion of infection to death. Uninfected women enter into the transmission module and transition into the disease module if they acquire the AIDS virus.

In the model constructed by Walensky and colleagues, the impact of pre-exposure prophylaxis in the transmission module was calculated by reducing incidence rates downwards by $39 \%$ (the authors used $39 \%$ as an estimate of the protective efficacy because this is the value estimated by the CAPRISA 004 trial that examined women who received pre-exposure prophylaxis using a tenofovir disoproxil fumarate-based vaginal microbicide in South Africa). Subsequently, the number of HIV infections averted was converted into an estimate of the cost per year of life gained using the parameters of a simulated cohort in the CEPAC-international disease progression module where the parameters were obtained from several South African studies. In particular, the authors used an estimate of \$0.32/dose (applicator and gel) and assumed that two doses per act were used (before and after the act) and that the mean number of acts per woman per month was 7.2. Using a simulated cohort of HIV-infected South African women, the authors found the incremental cost-effectiveness ratio was $\$ 2,700$ (2010 US\$) per year of life saved for the pre-exposure prophylaxis program using tenofovir disoproxil fumarate-based vaginal microbicide.

A group of researchers at the Centres for Disease Control and Prevention (CDC) [54] recently developed a dynamic compartmental model of the cost effectiveness of an HIV pre-exposure prophylaxis program for MSM in an urban setting. They obtained epidemic data for New York City and assumed that the pre-exposure prophylaxis program reduces the incidence of HIV by $44 \%$ among the general population of MSM and $73 \%$ among highly adherent participants, defined as those who took $90 \%$ of the medication at the appropriate time.

This model assumes that oral tenofovir disoproxil fumarate and emtricitabine cost $\$ 8,000$ a year, and the authors calculated that the cost of preventing an HIV infection was $\$ 870,590$ and that the cost per QALY gained was \$570,273 (2010 US\$). For persons who were highly adherent, the cost of preventing an HIV infection was $\$ 631,971$ and the cost per QALY gained was $\$ 353,739$ per person. These costs were high, primarily because the estimated increase in QALYs attributable to the pre-exposure prophylaxis program was quite small ( $<2$ QALYs) and the benefits were discounted at $3 \%$ per year and these benefits materialize many years in the future.

\section{Discussion}

Several cost-effectiveness studies of pre-exposure prophylaxis for HIV have been discussed in this manuscript, 
and differences in estimates of the cost effectiveness of pre-exposure prophylaxis programs for HIV infection vary widely. One reason for this is the variation in the cost of drugs in various countries. For example, Walensky and colleagues [49] estimated the cost of drugs for a tenofovir disoproxil fumarate-based vaginal microbicide was $\$ 55$ a year per person in South Africa (2010 US\$), and Juusola and colleagues [53] estimated the cost of drugs for an oral tenofovir disoproxil fumarate-based pre-exposure prophylaxis program was $\$ 15,589$ a year per person in the US (2010 US\$).

If the cost of drugs in South Africa were similar to the cost of drugs in the US, the cost per QALY associated with a tenofovir disoproxil fumarate-based vaginal microbicide pre-exposure prophylaxis program for HIV [49] would increase from $\$ 2,700$ to a figure closer to $\$ 270,000$. Such a program would not be cost effective in South Africa or in the US.

In the US, programs with costs per QALY less than $\$ 50,000$ are generally considered to be cost effective [55, 56]. The cost of Truvada is at least $\$ 10,000$ a year in the US (2010 US\$), and it is available in many poor nations for substantially less. In fact, Gilead Sciences Inc. indicate that a generic version of Truvada is available in more than 100 countries at prices as low as $\$ 108$ a year under provisions with 12 generic partners in India $[57,58]$. Thus, if the cost of Truvada were the same in the US as in many lessdeveloped nations, the cost per QALY in the three costeffectiveness studies cited in Table 2 would be reduced to a figure far less than the figure in Table 2 and each of these three pre-exposure prophylaxis programs for HIV would be cost effective.

The variation in the price of drugs in different countries is widely known, especially when it involves comparisons involving countries with low incomes and countries with high incomes. However, there are wide variations in the prices paid for antiretroviral drugs between countries with similar levels of development. Indeed, a study by Waning and colleagues [59] revealed that there are many antiretroviral drug purchases where the cost of drugs varied by tenfold between countries with low per capita incomes.

Although there are wide variations in estimates of the cost effectiveness for HIV pre-exposure prophylaxis programs across nations that in part are due to variations in the cost of drugs, there is considerable variation in estimates of the cost effectiveness for HIV pre-exposure prophylaxis programs in the US. In particular, Desai and colleagues [50] estimated that the cost per QALY saved for a preexposure prophylaxis program for MSM in the US was \$31,970 (2007 US\$), and Paltiel and colleagues [48] estimated the cost per QALY saved for a pre-exposure prophylaxis program for MSM in the US was \$298,000 (2006 US\$). Subsequently, Juusola and colleagues [53] estimated the cost per QALY saved for a pre-exposure prophylaxis program for MSM in the US to be $\$ 172,000$ (2010 US\$).

These differences reflect both the manner in which each study estimated the impact of pre-exposure prophylaxis on the number of HIV infections and differences in the timeframes over which the impact of the programs are evaluated. For example, Desai and colleagues [50] developed a risk-based HIV transmission model to analyze a 5-year pre-exposure prophylaxis program, while Juusola and colleagues [53] assume that their pre-exposure prophylaxis program was operational for 20 years.

In the study by Desai and colleagues [50], the authors adjusted coefficients in their model to produce measures of incidence and prevalence that coincided with data obtained from the CDC and the New York City Department of Health and Mental Hygiene. Then the number of HIV infections prevented by the chemoprophylaxis program was derived by comparing the number of infections in the absence of pre-exposure prophylaxis to estimates of the number of HIV infections in the presence of pre-exposure prophylaxis over a 5-year time period.

The model used by Juusola and colleagues [53] took into account the number of partners, number of sexual acts, types of sexual acts, viral load of each partner, and whether an individual adjusts his behaviour based on his assumptions about his partner. The length of duration of the relationship and the risk class of the infected partner also affected the HIV infection rates. This model incorporated the chemoprophylaxis program by adjusting a number of factors, including the estimated number of partners, the probability of transmission per sexual contact, and the probability of condom use.

An alternative approach to assessing the impact of HIV pre-exposure prophylaxis on the likelihood of HIV transmission was employed by Paltiel and colleagues [48] who employed an individual-level simulation to estimate the number of HIV infections. The impact of pre-exposure prophylaxis was incorporated by assuming an efficacy rate of pre-exposure prophylaxis of $50 \%$ in a high-risk population of MSM where the incidence rate of HIV (i.e. mean cases per 100 person-years incidence) was estimated to be $1.6 \%$. This approach is based on data from several clinical trials, indicating that uninfected persons in the target population experience about one-half the number of HIV infections as similar individuals in the control arm of the trial (i.e. persons taking placebos), and that estimates of the incidence of HIV in prior time periods are good approximations for the current time period.

If available, analysts may use data about the number of new HIV cases (i.e. HIV incidence) that occurred in the previous time period to estimate the number of HIV infections that would have occurred had the prevention program under study not been implemented. Yet, such data 
may not be available, in which case the analyst must use some other incidence rate. At a minimum, analysts should explain how they came up with their estimate of HIV incidence in the absence of the pre-exposure prophylaxis program under study.

Moreover, it is important for analysts to emphasize the inexactitude of their findings, and it is essential for policymakers and others who use these results to recognize that different assumptions about the patterns of sexual behaviour of MSM may produce very different estimates of the cost effectiveness of pre-exposure prophylaxis.

It is also possible that efficacy estimates derived in clinical trials in countries in Africa, Asia, and South America are not good estimates of efficacy of similar interventions in the US. Indeed, only one of the four clinical trials in Table 1 (iPrEx) included any individuals from the US, and only $3 \%$ of the 2,499 individuals in the iPrEx trial were from the US site in Boston.

Differences in estimates of the cost effectiveness of preexposure prophylaxis programs against HIV also reflect various approaches for translating a reduction in the number of infected individuals into an estimate of the increase in the number of QALYs gained. Because there is little consensus about how a reduction in one case of HIV disease translates into an increase in the number of QALYs (e.g. Desai and colleagues [50] estimate that a reduction in one case of HIV translates into a gain of 6.95 QALYs, while Paltiel and colleagues [48] estimated that a reduction in one case of HIV translated into a gain of only 1.7 QALYs), it makes sense to compare cost-effectiveness studies of pre-exposure prophylaxis against HIV using the number of HIV cases averted as the unit of effectiveness.

One method of addressing the uncertainty regarding estimates of the cost effectiveness of pre-exposure prophylaxis programs derived using mathematical models of HIV transmission is sensitivity analysis. Analysts may present information about the findings of the study using different values for important parameters about which there is relatively little solid data (e.g. number of sexual partners, viral load of partners, use of a condom, and type of sexual contact). In this way, analysts may convey information about how the conclusions of the study are effected by changing assumptions and this will inform the reader about the robustness of the model. In addition, it will indicate which assumptions are most critical and this may encourage future empirical studies to address the parameters of interest.

\section{Final Remarks}

During the first two decades of the HIV epidemic, significant medical progress was made in identifying and tracking the spread of this epidemic across the US. Although great strides were also made in the treatment of HIV, little progress was made in preventing its spread. Each year an estimated 50,000 persons are infected with HIV in the US [7, 53] and this number has remained virtually the same for the past decade.

Evidence from multinational randomized clinical trials has shown that antiretroviral therapy reduces the rate of HIV transmission by decreasing the vulnerability of highrisk individuals without HIV (i.e. those who have sexual contact with a person with HIV disease or share a needle with an individual with HIV disease). Yet, up to this point, there is no evidence showing that increases in the number of uninfected persons receiving antiretroviral therapy has led to a reduction in the number of persons diagnosed with HIV in the US.

It is not feasible to obtain evidence from randomized clinical trials of the cost effectiveness of pre-exposure prophylaxis because of the complex logistics, elevated costs, and ethical considerations involved with conducting trials in large populations over a significant period of time using a drug regimen that has already been shown to reduce a person's probability of becoming infected. The absence of data from randomized clinical trials has necessitated the use of mathematical models to conduct economic evaluations of health interventions. Indeed, mathematical models of HIV transmission are a valuable method of assessing the effects of pre-exposure prophylaxis because the indirect effect of pre-exposure prophylaxis may not be apparent for several years (i.e. a reduction in the number of people infected in a population in one time period lowers the likelihood of HIV transmission in forthcoming time periods).

Nevertheless, the development and calibration of mathematical models that reflect the impact of HIV prevention programs is challenging because there is rarely enough good data available to calibrate and compare different models. It is, however, true that clinical trial data may be used to construct and then to test a specific model but the analysts who have estimated the cost effectiveness of pre-exposure prophylaxis are not those who have conducted the clinical trials of pre-exposure prophylaxis. Moreover, clinical trial data may not include information about important determinants of HIV transmission (e.g. number of sexual contacts, proportion of contacts using a condom, reasonableness of the proportional sampling assumption, number of partners, probability of acquiring HIV per contact) and they rarely include data on the cost of services provided to participants (e.g. physician visits, medications, laboratory tests, and hospital care).

In addition, it is not clear how well estimates of adherence rates in clinical trials predict adherence rates in programs available to whole populations. This issue is 
important because randomized clinical trials show that persons with poor adherence rates are less likely to benefit from pre-exposure prophylaxis programs. In sum, the lack of good information about important parameters makes it difficult to construct good mathematical models of HIV transmission and to validate these models.

Indeed, some models are calibrated using available data on the number of new HIV cases in a specific geographic area. For example, the model used in the study by Desai and colleagues [50] was calibrated using data from CDC and the New York City Department of Health and Mental Hygiene. In this situation, the prevalence and incidence of HIV among MSM stratified by age derived using the model was used to adjust the parameters in the model so that its forecasts were reasonably close to observed levels. It is still uncertain, however, how well the predictions derived for the impact of a pre-exposure prophylaxis program are using this model.

Even so, the thinking among health care experts has changed and many policy makers now believe that there should be greater emphasis on initiating HIV treatment as a means by which to prevent the transmission of HIV infection in the US [60]. There are some who believe that simply stepping up the treatment of people with HIV will eventually reduce the spread of HIV in the US [12].

In a recent publication, the WHO [17] stressed the need for research about the best ways to scale up pre-exposure prophylaxis programs for different risk groups in countries in different stages of development. Indeed, it remains unclear whether or not the uptake of antiretroviral therapy by vulnerable uninfected persons will ever result in impressive reductions in new HIV infections in the US.

In a 2012 article, Mermin and Fenton [61] stated that:

The United States has an opportunity to shift from supporting hundreds of different HIV prevention approaches to objectively assessing current HIV strategies, focusing on more cost-effective activities, and conducting research that will establish the groundwork for the future.

Yet, conducting research on the cost effectiveness of pre-exposure prophylaxis programs has proved difficult and findings from these vary widely. One reason for this is the lack of reliable information about the patterns of sexual behaviour and drug use of populations where pre-exposure prophylaxis programs are likely to be implemented. Analysts estimate risk-based models of HIV transmission which require detailed information about the sexual patterns of MSM and about the networks of drug sharing among injection drug users.

Another reason is that these studies translate the reduction in number of HIV cases into an increase in the number of QALYs gained, and there is little agreement about how to estimate the impact of HIV on longevity. Yet, it is reasonable for those who conduct cost-effectiveness studies of pre-exposure prophylaxis programs to compare such programs using the cost per HIV infection averted. Use of the cost per HIV infection averted instead of cost per QALY gained obviates the need to convert the cost per HIV case averted into the cost per QALY gained. Nonetheless, it is evident that the high cost of pre-exposure prophylaxis and uncertainty about the coverage policies of insurers have hindered the diffusion of this strategy in the US [62].

One widely cited source of information about the impact of pre-exposure prophylaxis on the number of infections among MSM is the iPrEx trial [13]; this trial provides a glimpse of the costs involved in implementing a large-scale pre-exposure prophylaxis program aimed at reducing the number of new infections among MSM. In the iPrEx trial there were 1,250 MSM who received Truvada and the same number who received a placebo. There were 64 new infections in the placebo group and 36 in the Truvada group, which implies that providing pre-exposure prophylaxis to 1,250 uninfected MSM resulted in a reduction in 28 infections. Since the median observed time for participants in this trial was 1.2 years (here, it is assumed that the arithmetic mean and the median are comparable), it may be inferred that placing 1,250 uninfected MSM on Truvada for 1 year reduces the number of infections by 23 . The cost of providing Truvada to persons in the US is at least $\$ 10,000$ per year, which suggests that the cost of averting one case of HIV is at least $\$ 543,000[(1,250 \times \$ 10,000) / 23]$.

The high cost of Truvada in the US also suggests that the cost of a large-scale pre-exposure prophylaxis program could easily cost billions of dollars. For example, Juusola and colleagues [53] estimated that the number of MSM aged 13-64 years in the US was 4.3 million. Thus, if a preexposure prophylaxis program enrolled 4.3 million persons it would cost about $\$ 43$ billion. This, of course, is an upper bound. Indeed, it is doubtful that any pre-exposure prophylaxis program will treat more than a small proportion of this population given the fiscal constraints on government payers (e.g. Medicaid, Medicare, and the Department of Veterans Affairs) and the reluctance of private insurers to commence a large-scale program that provides expensive prophylactic drugs to uninfected individuals.

The diversity in methods, assumptions, and models used to estimate the economic and health-related outcomes of interventions such as pre-exposure prophylaxis programs for HIV makes it difficult to compare findings across studies. This obstacle motivated the development of guidelines for economic evaluations of health interventions. In particular, the International Society for Pharmacoeconomics and Outcomes Research (ISPOR) produced reporting guidelines to facilitate the comparison of study 
findings. These guidelines include 24 items grouped into six categories (Title and Abstract, Introduction, Methods, Results, Discussion, and Other). The Methods category is the largest and includes 14 items (target population, setting and location, study perspective, type of intervention, time horizon, discount rate, choice of health outcomes, measurement of effectiveness, measurement and valuation of preference-based outcomes, costs of intervention, price data, choice of model, assumptions, and analytic methods). These guidelines are referred to as the Consolidated Health Economic Evaluation Reporting Standards (CHEERS) [63] and their use should help mitigate this problem. However, the value of these guidelines depends on their widespread use and it will be some time before this is known.

Acknowledgments No sources of funding were used to assist in the preparation of this study. The author has no conflicts of interest that are directly relevant to the content of this study.

\section{References}

1. Mathers BM, Degenhardt L, Bucello C, Lemon J, Wiessing L, Hickman M. Mortality among people who inject drugs: a systematic review and meta-analysis. Bull World Health Organ. 2013;91(2):102-23.

2. Schneider G, Juday T, Wentworth C 3rd, Lanes S, Hebden T, Seekins D. Impact of health care payer type on HIV stage of illness at time of initiation of antiretroviral therapy in the USA. AIDS Care. 2013;25(11):1470-6.

3. Dilernia DA, Monaco DC, Cesar C, Krolewiecki AJ, Friedman SR, Cahn P, Salomon H. Estimation of HIV-testing rates to maximize early diagnosis-derived benefits at the individual and population level. PLoS One. 2013;8(1):e53193.

4. Nakagawa F, Lodwick RK, Smith CJ, Smith R, Cambiano V, Lundgren JD, Delpech V, Phillips AN. Projected life expectancy of people with HIV according to timing of diagnosis. AIDS. 2012;26(3):335-43.

5. Hall HI, Song R, Rhodes P, et al. Estimation of HIV incidence in the United States. JAMA. 2008;300:520-9.

6. US Centers for Disease Control and Prevention. Interim guidance for clinicians considering the use of preexposure prophylaxis for the prevention of HIV infection in heterosexually active adults. MMWR Morb Mortal Wkly Rep. 2012;61(31):586-9.

7. Prejean J, Song R, Hernandez A, Ziebell R, Green T, et al. Estimated HIV incidence in the United States, 2006-2009. PLoS ONE. 2011;6(8):e17502.

8. Sullivan PS, Carballo-Diéguez A, Coates T, Goodreau SM, McGowan I, Sanders EJ, Smith A, Goswami P, Sanchez J. Successes and challenges of HIV prevention in men who have sex with men. Lancet. 2012;380(9839):388-99.

9. Cohen MS, Chen YQ, McCauley M, Gamble T, Hosseinipour $\mathrm{MC}$, et al. Prevention of HIV-1 infection with early antiretroviral therapy. N Engl J Med. 2011;365:493-505.

10. Amico KR, McMahan V, Goicochea P, Vargas L, Marcus JL, Grant RM, et al. Supporting study product use and accuracy in self-report in the iPrEx study: next step counseling and neutral assessment. AIDS Behav. 2012;16(5):1243-59.

11. Cremin I, Alsallaq R, Dybul M, Piot P, Garnett G, Hallett TB. The new role of antiretrovirals in combination HIV prevention: a mathematical modelling analysis. AIDS. 2013;27(3):447-58.
12. HIV Modelling Consortium Treatment as Prevention Editorial Writing Group. HIV treatment as prevention: models, data, and questions-towards evidence-based decision-making. PLoS Med. 2012;9(7):e1001259.

13. Grant RM, Lama JR, Anderson PL, McMahan V, Liu AY, et al. Preexposure chemoprophylaxis for HIV prevention in men who have sex with men. N Engl J Med. 2010;363:2587-99.

14. Cohen J. Breakthrough of the year: HIV treatment as prevention. Science. 2011;34:1628-9.

15. Underhill K. Paying for prevention: challenges to health insurance coverage for biomedical HIV prevention in the United States. Am J Law Med. 2012;38(4):607-66.

16. Gomez GB, Borquez A, Case KK, Wheelock A, Vassall A, Hankins C. The cost and impact of scaling up pre-exposure prophylaxis for HIV prevention: a systematic review of costeffectiveness modelling studies. PLoS Med. 2013;10(3): e1001401.

17. World Health Organization. Guidance on pre-exposure oral prophylaxis (PrEP) for serodiscordant couples, men and transgender women who have sex with men at high risk of HIV: recommendations for use in the context of demonstration projects. Geneva, Switzerland; 2012.

18. Krakower D, Mayer KH. What primary care providers need to know about preexposure prophylaxis for HIV prevention: a narrative review. Ann Intern Med. 2012;157(7):490-7.

19. Okwundu CI, Uthman OA, Okoromah CA. Antiretroviral preexposure prophylaxis (PrEP) for preventing HIV in high-risk individuals. Cochrane Database Syst Rev. 2012;(7):CD007189.

20. Karim AQ, Abdool Karim SS, Frohlich JA, Grobler AC, Baxter $\mathrm{C}$, et al. Effectiveness and safety of tenofovir gel, an antiretroviral microbicide, for the prevention of HIV infection in women. Science. 2010;329:1168-74.

21. Baeten JM, Donnell D, Ndase P, Mugo NR, Campbell JD, et al. Antiretroviral prophylaxis for HIV prevention in heterosexual men and women. N Engl J Med. 2012;367:399-410.

22. Thigpen MC, Kebaabetswe PM, Paxton LA, Smith DK, Rose CE, et al. Antiretroviral preexposure prophylaxis for heterosexual HIV transmission in Botswana. N Engl J Med. 2012;367:423-34.

23. Van Damme L, Corneli A, Ahmed K, et al. Preexposure prophylaxis for HIV infection among African women. N Engl J Med. 2012;367:411-22.

24. Microbicide Trials Network. Backgrounder: VOICE—Vaginal and Oral Interventions to Control the Epidemic. National Institute of Allergy and Infectious Diseases (NIH), 18 Nov 2012. (http:// www.mtnstopshiv.org/news/studies/mtn003/backgrounder).

25. Vermund SH, Hayes RJ. Combination prevention: new hope for stopping the epidemic. Curr HIV/AIDS Rep. 2013;10(2):169-86.

26. Hankins CA, Dybul MR. The promise of pre-exposure prophylaxis with antiretroviral drugs to prevent HIV transmission: a review. Curr Opin HIV AIDS. 2013;8(1):50-8.

27. Stanley EA. Social networks and mathematical modeling. Connections. 2006;27(1):43-9.

28. Hyman JM, Stanley EA. Using mathematical models to understand the AIDS epidemic. Math Biosci. 1988;90:415-73.

29. Juusola JL, Brandeau ML, Long EF, Owens DK, Bendavid E. The cost-effectiveness of symptom-based and routine screening for acute HIV infection in men who have sex with men in the USA. AIDS. 2011;25:1779-87.

30. Vallabhaneni S, et al. Seroadaptive behavior: association with seroconversion among HIV-MSM. In: Nineteenth Conference on Retroviruses and Opportunistic Infections (CROI), Seattle [abstract 140]; 2012 (the abstract is available on the official conference website)

31. Baggaley RF, Fraser C. Modelling sexual transmission of HIV: testing the assumptions, validating the predictions. Curr Opin HIV AIDS. 2010;5:269-76. 
32. Curran JW, Jaffe HW, Hardy AM, Morgan WM, Selki RM, Dondero TJ. Epidemiology of HIV infection and AIDS in the United States. Science. 1988;239:610-6.

33. Hethcote HW, Van Ark JW. Epidemiological models for heterogeneous populations: proportionate mixing, parameter estimation, and immunization programs. Math Biosci. 1987;84:85-118.

34. Wiley JA, Herschkorn S, Padian N. Heterogeneity in the probability of HIV transmission per sexual contact. Stat Med. 1989;8:93-102.

35. Nonacs P, Kapheim KM. Modeling disease evolution with multilevel selection: HIV as a quasispecies social genome. J Evol Med. 2012;1:1.

36. Zintzaras E, Kowald A. A mathematical model of HIV dynamics in the presence of a rescuing virus with replication deficiency. Theory Biosci. 2011;130(2):127.

37. Yuan Y, Allen LJ. Stochastic models for virus and immune system dynamics. Math Biosci. 2011;234(2):84.

38. Mathieu E, Loup P, Dellamonica P, Daures JP. Markov modelling of immunological and virological states in HIV-1 infected patients. Biom J. 2005;47(6):834-46.

39. Hendriks JC, Satten GA, Longini IM, van Druten HA, Schellekens PT, Coutinho RA, van Griensven GJ. Use of immunological markers and continuous-time Markov models to estimate progression of HIV infection in homosexual men. AIDS. 1996;10(6):649-56.

40. Ruff CT, Ray SC, Kwon P, Zinn R, Pendleton A, Hutton N, Ashworth R, Gange S, Quinn TC, Siliciano RF, Persaud D. Persistence of wild-type virus and lack of temporal structure in the latent reservoir for human immunodeficiency virus type 1 in pediatric patients with extensive antiretroviral exposure. J Virol. 2002;76(18):9481-92.

41. Finzi D, Blankson J, Siliciano JD, et al. Latent infection of CD4+ $\mathrm{T}$ cells provides a mechanism for lifelong persistence of HIV-1, even in patients on effective combination therapy. Nat Med. 1999;5:512-7.

42. Manner IW, Trøseid M, Oektedalen O, Baekken M, Os I. Low nadir CD4 cell count predicts sustained hypertension in HIVinfected individuals. J Clin Hypertens (Greenwich). 2013; 15(2):101-6.

43. Kaufmann GR, Furrer H, Ledergerber B, Perrin L, Opravil M, Vernazza P, Cavassini M, Bernasconi E, Rickenbach M, Hirschel B, Battegay M, Swiss HIV Cohort Study. Characteristics, determinants, and clinical relevance of CD4 T cell recovery to $<500$ cells/microL in HIV type 1-infected individuals receiving potent antiretroviral therapy. Clin Infect Dis. 2005;41(3):361-72.

44. Baraboutis IG, Papastamopoulos V, Georgiou O, Skoutelis AT. Revertant multiresistant HIV in chronically infected drug naïve patients: when baseline resistance testing is not enough. Int J STD AIDS. 2007;18(10):722-4.

45. US Federal Funding for HIV/AIDS. The President's FY 2012 Budget, Kaiser Family Foundation HIV/AIDS Policy Fact Sheet; 2011.

46. Kates J. Spending on the HIV/AIDS epidemic: trends in U.S. spending on HIV/AIDS, Menlo Park, California. The Henry J. Kaiser Family Foundation; 2002.

47. Sanders GD, Bayoumi AM, Sundaram V, Bilir SP, Neukermans CP, Rydzak CE, Douglass LR, Lazzeroni LC, Holodniy M, Owens DK. Cost-effectiveness of screening for HIV in the era of highly active antiretroviral therapy. $\mathrm{N}$ Engl J Med. 2005;352(6):570-85.

48. Paltiel AD, Weinstein MC, Kimmel AD, et al. Expanded screening for HIV in the United States: an analysis of costeffectiveness. N Engl J Med. 2005;352:586-95.

49. Walensky RP, Park JE, Wood R, Freedberg KA, Scott CA, Bekker LG, Losina E, Mayer KH, Seage GR 3rd, Paltiel AD. The cost-effectiveness of pre-exposure prophylaxis for HIV infection in South African women. Clin Infect Dis. 2012;54(10):4-13.

50. Desai K, Sansom SL, Ackers ML, Stewart SR, Hall HI, et al. Modeling the impact of HIV chemoprophylaxis strategies among men who have sex with men in the United States: HIV infections prevented and cost-effectiveness. AIDS. 2008;22:1829-39.

51. Paltiel AD, Freedberg KA, Scott CA, Schackman BR, Losina E, et al. HIV preexposure prophylaxis in the United States: impact on lifetime infection risk, clinical outcomes, and cost-effectiveness. Clin Infect Dis. 2009;48:806-15.

52. Freedberg KA, Losina E, Weinstein MC, Paltiel AD, Cohen CJ, Seage GR, Craven DE, Zhang H, Kimmel AD, Goldie SJ. The cost effectiveness of combination antiretroviral therapy for HIV disease. N Engl J Med. 2001;344(11):824-31.

53. Juusola JL, Brandeau ML, Owens DK, Bendavid E. The costeffectiveness of pre-exposure prophylaxis for HIV prevention in the United States in men who have sex with men. Ann Intern Med. 2012;156:541-50.

54. Koppenhaver RT, Sorensen SW, Farnham PG, Sansom SL. The cost-effectiveness of pre-exposure prophylaxis in men who have sex with men in the United States: an epidemic model. J Acquir Immune Defic Syndr. 2011;58:e51-2.

55. Farnham PG, Holtgrave DR, Sansom SL, Hall HI. Medical costs averted by HIV prevention efforts in the United States, 1991-2006. JAIDS. 2010;54(5):565-7.

56. Braithwaite RS, Meltzer DO, King JT Jr, Leslie D, Roberts MS. What does the value of modern medicine say about the $\$ 50,000$ per quality-adjusted life-year decision rule? Med Care. 2008;46(4):349-56.

57. Pierson R. WHO endorses use of HIV medicines for prevention. Chicago Tribune; 2012.

58. Keller SB, Smith DM. The price of tenofovir-emtricitabine undermines the cost-effectiveness and advancement of preexposure prophylaxis. AIDS. 2011;25(18):2308-10.

59. Waning B, et al. Temporal trends in generic and brand prices of antiretroviral medicines procured with donor funds in developing countries. J Generic Med. 2010;7(2):159-75.

60. Rayment M. Prevention of HIV-1 infection with early antiretroviral therapy. J Fam Plan Reprod Health Care. 2012;38:193.

61. Mermin J, Fenton KA. The future of HIV prevention in the United States. JAMA. 2012;308(4):347-8.

62. Williams B et al. Treatment as prevention: preparing the way. J Int AIDS Soc. 2010;14(1). http://www.avert.org/hiv-treatment-asprevention.htm\#sthash.QL8HClqI.dpuf.

63. Husereau D, Drummond M, Petrou S, Carswell C, Moher D, Greenberg D, Augustovski F, Briggs AH, Mauskopf J, Loder E, ISPOR Health Economic Evaluation Publication GuidelinesCHEERS Good Reporting Practices Task Force. Consolidated Health Economic Evaluation Reporting Standards (CHEERS). Value Health. 2013;16(2):231-50. 